\title{
Pathogenesis and Pulmonary Pathology in SARS-CoV-2 (COVID-19) Host Cell Invasion
}

\author{
Attapon Cheepsattayakorn ${ }^{1 *}$ and Ruangrong Cheepsattayakorn ${ }^{2}$ \\ ${ }^{1} 10^{\text {th }}$ Zonal Tuberculosis and Chest Disease Center, Chiang Mai, Thailand \\ ${ }^{2}$ Department of Pathology, Faculty of Medicine, Chiang Mai University, Chiang Mai, \\ Thailand
}

*Corresponding Author: Attapon Cheepsattayakorn, $10^{\text {th }}$ Zonal Tuberculosis and Chest Disease Center, Chiang Mai, Thailand.
Received: September 21, 2020

Published: September 30, 2020

(C) All rights are reserved by Attapon

Cheepsattayakorn and Ruangrong

Cheepsattayakorn.
Coronaviruses are enveloped and single-stranded ribonucleic acid (RNA) viruses of approximately $30 \mathrm{~kb}$ with infections of various host species. SARS-CoV-2 (COVID-19) are divided into four genera; $\alpha, \beta, \gamma$ and $\delta$ based on their genomic structure. Alpha and beta coronaviruses infect only mammals. SARS-CoV-2 (COVID-19), SARS-CoV and Middle-East-Respiratory-Syndrome coronavirus (MERS-CoV) are classified to $\beta$ coronaviruses.

In the host, the life cycle of coronavirus consists of 5 steps: 1 ) attachment, 2) penetration, 3) biosynthesis, 4) maturation and 5) release. Once viruses attach host receptors, they penetrate host cells by membrane fusion, particularly type II pneumocytes, including by endocytosis. Then viral RNA enters the host's nucleus for biosynthesis (viral proteins synthesis). New viral particles are produced (maturation) and released.

Coronaviruses consist of four structural proteins; spike (S), membrane (M), envelop (E), and nucleocapsid (N). Spike protein of coronaviruses which determines the diversity of coronaviruses and host tropism is composed of a transmembrane trimetric glycoprotein protruding from the viral surface. Spike protein comprises S1 functional subunit which is responsible for host cell receptor binding and S2 functional subunit which is responsible for viral and cellular membrane fusion. Structural and functional studies demonstrated that the spike protein the of coronaviruses can bind to angiotensin converting enzyme 2 (ACE2), a functional receptor for SARS-CoV. ACE2 expression is high in lung (high expression on lung epithelial cells), heart, ileum, and kidney. Further studies are needed for additional SARS-CoV-2 (COVID-19) binding targets.

After binding of SARS-CoV-2 (COVID-19) to the host protein, protease cleavage is underwent by the spike protein. The spike protein of SARS-CoV-2 (COVID-19) and MERS-CoV are activated and primed as a two-step sequential protein cleavage at the S1/ S2 cleavage site, a position adjacent to a fusion peptide within the
S2 subunit site. Following the cleavage at the S1/S2 cleavage site, S1 and S2 subunits remain non-covalently bound and the distal S1 subunit leads to the stabilization of the membrane-anchored S2 subunit at the pre-fusion state. Presumably activation of the spike protein for membrane fusion through irreversible and conformational changes is due to subsequent cleavage at the S2 site. Existence of furin cleavage site ("RPPA" sequence) at the S1/S2 site is the unique characteristics of SARS-CoV-2 (COVID-19) among coronaviruses. During biosynthesis, the S1/S2 site of SARS-CoV-2 (COVID-19) is entirely subjected to cleavage in a drastic contrast to SARS-CoV spike protein that is incorporated without cleavage. Although the S1/S2 site is subjected to cleavage by other protease, such as cathepsin $\mathrm{L}$ and transmembrane protease serine 2 (TMPRSS2), making SARS-CoV-2 (COVID-19) very pathogenic is by the expression of furin.

Antigen presentation through dendritic cells (DCs) is demonstrated by $\mathrm{T}$-cell mediated responses against coronaviruses and phagocytization of virus-infected-apoptotic epithelial cells can be contributed by macrophage leading to antigen presentation to $\mathrm{T}$ cells. The expression of ACE2 on (splenic) dendritic cells and pulmonary alveolar macrophages is present but limited, based on the Immunological Genome database (http://rstats.immgen.org). DCs and macrophages may be primarily infected with virus. SARS-CoV-2 (COVID-19) uses another protein to bind to antigen presenting cells (ACPs) or not should be investigated. These ACPs move to the draining lymph nodes to present viral antigens to T cells. Dendritic-cell specific intercellular adhesion molecule-3-grabbing nonintegrin (DC-SIGN, the high expression on dendritic cells and macrophages) and DC-SIGN-related protein (DC-SIGNR, L-SIGN) can be bound by SARS-CoV in addition to ACE2 binding. CD4+ T cells can activate B cells to promote the production of virus-specific antibody, whereas CD8+ T cells can kill viral infected cells. 
Patients with severe COVID-19 demonstrated lymphopenia, especially in peripheral blood T cells and increased plasma concentrations of granulocyte-colony stimulating factor (G-CSF), interleukin (IL)-6, IL-10, macrophage inflammatory protein (MIP)-1 $\alpha$, monocyte chemoattractant protein 1 (MCP-1) and tumor necrosis factor (TNF)- $\alpha$. The higher levels of IL- 6 are, the more severe conditions the COVID-19 patients are in. Higher expression of CD38, CD44, and CD69 is demonstrated in COVID-19 patients with activation of CD4+ and CD8+ T cells. T cells exhaustion that could have led to the progression of COVID-19 is indicated by higher percentage of checkpoint receptor Tim-3+ PD-1+ subsets in CD4+ and CD8+ T cells. Another marker for T cells exhaustion is elevation of NK group 2 member A (NKG2A) on CD8+ T cells.

In severe COVID-19 patients, there are aberrant pathogenic CD4+ T cells with co-expressing interferon (IFN)- $\gamma$ and granulocyte-macrophage colony-stimulating factor (GM-CSF). Significant decrease in circulating $\mathrm{T}$ cells, the majority of infiltrating adaptive immune cells are primary cytotoxic CD8+ T cells. CD4+ T cells are also pathological cytotoxic T cells found in severe COVID-19 patients with lung injury. These pathological CD4+ T cells release circulating monocytes responding to GM-CSF. Significant higher percentage of CD14+CD16+ inflammatory subsets are also identified in COVID-19 patients, but they are seldom exist in health individuals. Acceleration of the progression of systemic inflammatory response is demonstrated by high IL- 6 expression in these inflammatory CD14+CD16+ inflammatory monocytes. GM-CSF, a response to virus infection can assist in differentiation of innate immune cells augment $\mathrm{T}$ cell function, but GM-CSF can trigger tissue damage at excess. Previous experimental autoimmune encephalomyelitis (EAE) models in adults revealed that GM-CSF+IFN- $\gamma+\mathrm{CD} 4+\mathrm{T}$ cells were demonstrated upon strong $\mathrm{T}$ cell receptor (TCR) responses, whereas CD8+ T cells expressing GM-CSF were identified at higher percentage and secreted IL-6. Neutrophils, the majority of innate immune cells can induce lung injury.

SARS-CoV-infected lung epithelial cells can produce IL-8, a wellknown chemoattractant for neutrophils and $\mathrm{T}$ cells, in addition to IL-6 production. DCs are located underneath the epithelium and macrophages reside at the apical side of the epithelium, whereas epithelial cells, pulmonary alveolar macrophages, and dendritic cells (DCs) are the three main components for innate immunity in human airway. The lungs of severe COVID-19 patients demonstrate infiltration of a large number of inflammatory cells. Due to high ACE2 expression on the apical side of lung epithelial cells in the alveolar space, SARS-CoV-2 (COVID-19) can enter and destroy lung epithelial cells. Significant ACE2 expression on innate lymphoid cells (ILC)2, ILC3 and endothelial cells is also demonstrated. NK cells, a member of ILC1 constitute a majority of pulmonary ILCs, approximately $95 \%$, whereas ILC2 and ILC3 are responsible for mucous homeostasis. Nevertheless, there is a very limited knowledge of ILC2- and ILC3-involved coronavirus infection. Pulmonary endothelial cells represent one third of the lung cells. Endothelial function includes promotion of anti-aggregation, fibrinolysis, and vasodilatation. Due to a significant role playing in thrombotic regulation, hypercoagulable profiles that are demonstrated in severe COVID-19 patients likely suggest significant endothelial injury. Pulmonary endothelial injury can facilitate viral invasion through abnormal microvascular permeability. Pulmonary thrombosis and embolism accompanying elevation of d-dimer and fibrinogen levels have been demonstrated in severe COVID-19. The clinical features of SARS-CoV-2-infected patients vary from minimal symptoms to severe respiratory failure with multiple organ failure, in addition to pulmonary thrombosis and embolism. Computed tomography (CT) of the chest in COVID-19 patients reveals the characteristic pulmonary ground glass opacification even in the asymptomatic patients.

The hypotheses of the difference of pathophysiology between children and adults in COVID-19 are as the following: 1) The expression level of ACE2 may differ between children and adults, 2) Children qualitatively and differently respond to the SARS-CoV-2 (COVID-19) virus, compared to adults, and 3) Competition and growth limitation of SARS-CoV-2 (COVID-19) virus can be contributed by the simultaneous presence of other viruses in the pulmonary and airway mucosa of the young children.

In conclusion, further studies on understanding the roles of ILC1, ILC2, ILC3, and the difference in response to SARS-CoV-2 (C) OVID-19) infection between children and adults are urgently needed to develop efficient targeted therapies.

\section{Assets from publication with us}

- Prompt Acknowledgement after receiving the article

- Thorough Double blinded peer review

- Rapid Publication

- Issue of Publication Certificate

- High visibility of your Published work

Website: www.actascientific.com/

Submit Article: www.actascientific.com/submission.php

Email us: editor@actascientific.com

Contact us: +919182824667 